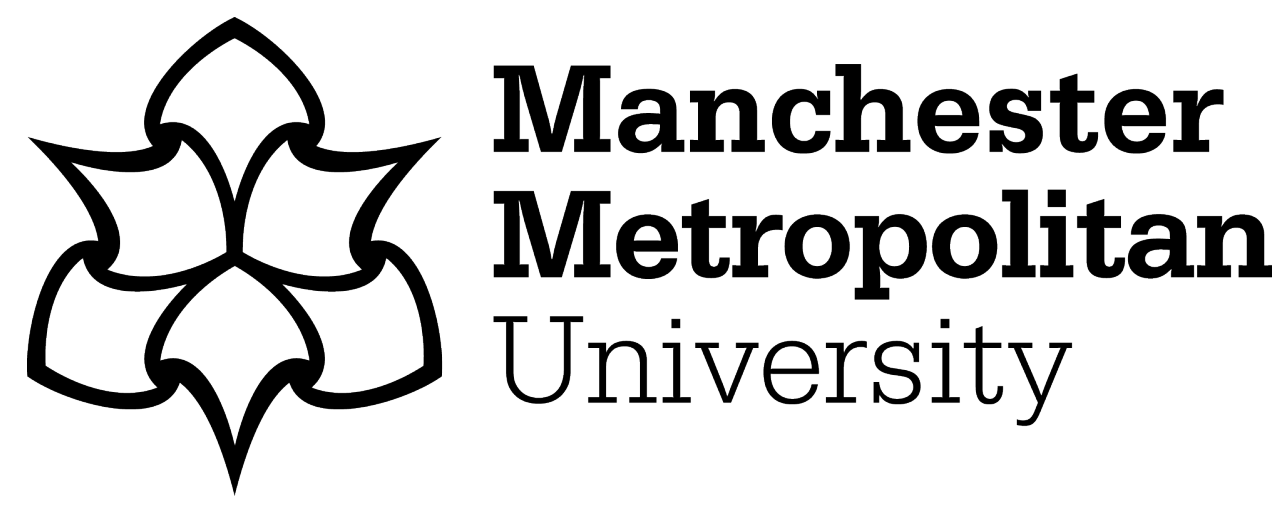

Green, D, Skeoch, S, Alexander, MY, Kalra, PA and Parker, B (2017) The Association of Baseline and Longitudinal Change in Endothelial Microparticle Count with Mortality in Chronic Kidney Disease. Nephron, 135 (4). pp. 252260. ISSN 1660-8151

Downloaded from: https://e-space.mmu.ac.uk/620901/

Version: Accepted Version

Publisher: Karger

DOI: https://doi.org/10.1159/000452344

Please cite the published version 


\title{
The Association of Baseline and Longitudinal Change in Endothelial Microparticle Count with Mortality in Chronic Kidney Disease
}

\author{
Darren Green $^{\mathrm{a}, \mathrm{e}}$ Sarah Skeoch ${ }^{\mathrm{b}}$ M. Yvonne Alexander ${ }^{\mathrm{c}}$ \\ Philip A. Kalra ${ }^{a, e}$ Ben Parker ${ }^{b, d}$ \\ ${ }^{a}$ Institute of Population Health, and ${ }^{b}$ Centre for Musculoskeletal Research, Institute of Inflammation and Repair, \\ University of Manchester, ${ }^{C}$ Healthcare Science Research Centre, Manchester Metropolitan University, and \\ ${ }^{d}$ NIHR Manchester Musculoskeletal Biomedical Research Unit, Central Manchester University Hospital NHS \\ Foundation Trust and Manchester Academic Health Science Centre, Manchester, and 'Vascular Research Group, \\ Salford Royal Foundation Trust, Salford, UK
}

\section{Key Words}

Chronic kidney disease - Endothelial microparticle .

Cardiovascular disease $\cdot$ Survival

\begin{abstract}
Background: Chronic kidney disease (CKD) is associated with a unique milieu of vascular pathology, and effective biomarkers of active vascular damage are lacking. A candidate biomarker is the quantification of circulating endothelial microparticles (EMPs). This study observed baseline and longitudinal EMP change ( $\delta E M P)$ and established the association of these with all-cause mortality and cardiovascular events in CKD. Method: An observational study in adults with CKD (estimated glomerular filtration rate [eGFR] $<60$ $\mathrm{mL} / \mathrm{min} / 1.73 \mathrm{~m}^{2}$ ). EMPs were quantified by flow cytometry of platelet poor plasma in 2 samples 12 months apart and categorised as EMP if AnnexinV+/CD31+/CD42b- EMPs were compared between primary renal diagnoses, and correlations between EMP/ठEMP and other parameters made. Adjusted hazard ratios (HRs) for time to all-cause mortality and cardiovascular events were calculated for log-transformed EMP and $\delta$ EMP using a Cox proportional hazard model. Results: There were 123 patients (age $63 \pm 11$ years,
\end{abstract}

systolic blood pressure $135 \pm 18 \mathrm{~mm} \mathrm{Hg}$, eGFR $32 \pm 16 \mathrm{~mL} /$ $\mathrm{min} / 1.73 \mathrm{~m}^{2}$ ). The median baseline EMP count was $144 / \mu \mathrm{L}$ (range 10-714/ $\mathrm{LL}$ ). EMPs were numerically the highest in autosomal dominant polycystic kidney disease (253 [41-610]). An increase in urine protein:creatinine ratio was associated with an increase in EMP (co-efficient 0.21, $p=0.02$ ). The adjusted HR for all-cause mortality for EMP was 8.20 (1.67-40.2, $p=0.01)$ and for $\delta E M P$ was $2.69(0.04-165, p=0.64)$. There was no association between EMP or $\delta E M P$ and cardiovascular events. Conclusion: Although EMP count was a significant marker of mortality risk, longitudinal change was not. This may reflect disease-specific EMP behaviour and the limitation of EMP as a generalised biomarker in CKD.

(c) 2017 S. Karger AG, Basel

\section{Introduction}

Chronic kidney disease (CKD) is associated with adverse cardiovascular outcome. Thirty-eight percent of dialysis patients have evidence of coronary artery disease [1], and the life expectancy of a 25-year-old dialysis patient is 12 years compared to 52 years in the general population [2]. 
It is generally agreed that this increased morbidity and mortality burden is not by way of "traditional" cardiovascular risk factors such as hyperlipidaemia and smoking. As a result, biomarkers of cardiovascular disease activity or prognosis in CKD are often different to those seen in the general population. For example, lipid-lowering therapy is less efficacious in $\mathrm{CKD}$ than the general population [3], and low serum lipids are more often a marker of malnutrition than of effective therapy in advanced CKD [4].

Non-atheromatous pathways to cardiovascular disease include chronic inflammation, hyperparathyroidism, and endothelial dysfunction [5-8]. CKD is associated with high circulating biomarkers of chronic inflammation, such as C-reactiveprotein(CRP)andinterleukin-6 [9]. However, these are not specific to vascular dysfunction. What may prove to be of greater clinical utility are emerging biomarkers of pathological vascular endothelial activity [10-12].

One potential candidate is a group of circulating vesicles known as endothelial microparticles (EMP). EMPs are membrane-bound anuclear vesicles shed by the vascular endothelium in response to a number of potential triggers such as shear stress or inflammation [13]. EMPs carry a number of activation and functional proteins, such as the angiogenic vascular endothelial growth factor and the vascular cell adhesion molecule (VCAM), which often reflect the underlying trigger that led to microparticle release [14]. Elevated EMPs have been associated with subclinical vascular damage including increased aortic pulse wave velocity (PWV), increased common carotid media thickness and endothelial dysfunction [15]. Elevated numbers of these particles have been reported in association with a number of diseases such as an increased risk of acute coronary syndrome and cardiovascular death [16], hypertension [17], pre-eclampsia [18] and metabolic syndrome [19]. EMPs also have prognostic potential having been shown to correlate with clinical response to therapy in systemic lupus erythematosus [20], and predict adverse events in otherwise stable coronary artery disease [16]. In CKD, higher circulating levels of EMP have been found in haemodialysis patients compared to pre-dialysis CKD, peritoneal dialysis and healthy controls [21-23]. In haemodialysis patients, EMPs and other microparticles have been shown to correlate with arterial vascular stiffness measured by PWV and augmentation index (AIx) [24].

No study appears to have longitudinally measured EMPs in CKD, nor correlated changes in EMPs with concurrent longitudinal changes in estimated glomerular filtration rate (eGFR). Alongside this, variation in levels and behaviour of EMPs between different primary renal diagnoses causing CKD are not understood. This is a vital consideration given that CKD is a common clinical manifestation of multiple disease entities rather than a singular disease state. Understanding any potential role of EMP as a biomarker in CKD may require disease-specific considerations.

The aims of this study, therefore, were to explore differences in EMP count and longitudinal change in EMP ( $\delta \mathrm{EMP})$ between different primary renal diseases, and to observe the association of absolute EMP count and $\delta$ EMP with (1) absolute and longitudinal changes in eGFR and other possible markers of CKD-associated cardiovascular risk such as hyperparathyroidism, inflammation and anaemia; (2) vascular stiffness as measured by AIx; and (3) longterm clinical outcome, particularly cardiovascular events.

\section{Method}

This was a sub-study of the Salford Kidney Study, a single-centre, longitudinal, observational study of patients with stage 3 to 5 CKD referred to an adult secondary care nephrology outpatient clinic $[25,26]$.

\section{Patients}

Patients were approached for inclusion in the study if aged $>18$ years and with eGFR $<60 \mathrm{~mL} / \mathrm{min} / 1.73 \mathrm{~m}^{2}$, and enrolled if written informed consent was gained. Patients were excluded from this sub-study if they had a history of any previous cardiovascular event including during the time between baseline and repeat EMP sampling (myocardial infarction, stroke, limb or gut ischaemia, coronary or peripheral revascularisation, hospitalisation for heart failure or symptomatic arrhythmia), or if they had a history of smoking. A target of 125 patients was then sought to include 25 patients in each of 5 primary renal disease categories (c-antineutrophil cytoplasmic antibody [cANCA] vasculitis, IgA nephropathy, diabetic nephropathy, hypertensive nephrosclerosis, autosomal dominant polycystic kidney disease [ADPKD]). These were chosen to cover inflammatory and non-inflammatory renal disease, and proteinuric and non-proteinuric diseases. The diagnoses were histological in cANCA and IgA disease, based on imaging in APKD, and either histological or presumed diagnoses in diabetic and hypertensive disease. In cases diagnosed as being diabetic nephropathy without biopsy, we necessitated the presence of proteinuria (urine protein:creatinine ratio $[\mathrm{uPCR}]>100 \mathrm{~g} / \mathrm{mol}$ ) and diabetic retinopathy. A diagnosis of hypertensive disease without biopsy was made on the basis of hypertension, evidence of endorgan damage elsewhere such as left ventricular hypertrophy on echocardiography, and the absence of urinary sediment or evidence of an alternative diagnosis. This, we acknowledge, is a less robust approach than using only biopsy-proven cases, but better reflects real-life practice. Patients with cANCA vasculitis or IgA nephropathy were included only if they were on maintenance immunosuppression and not initiation therapy, and if antibody was negative at the time of consent in the case of cANCA. Twenty-five 
consecutive patients with each primary disease who met these inclusion and exclusion criteria were then targeted for inclusion in this analysis.

\section{Clinical Parameters}

At the time of enrollment, patients were assessed for blood pressure (BP), demographic data, Karnofsky Performance Score, past medical history, prescribed medication, pulse wave analysis (PWA) and standard biochemical laboratory profile including MDRD calculated eGFR. An additional platelet poor plasma (PPP) citrate sample was collected and frozen at $-80^{\circ} \mathrm{C}$ for EMP quantification in batches. All laboratory samples, including PPP, were repeated at a second visit after 12 months. Patients were followed up for a minimum of 12 months after second visit and outcome data was collected by way of mortality data, including cause of death, sourced from the United Kingdom Office of National Statistics.

Pulse Wave Analysis

PWA was assessed by applanation tonometry using a Spygmo$\operatorname{Cor}^{\circledR}$ (AtCor Medical, West Ryde, NSW, Australia). The primary measure of aortic vascular stiffness in this study was AIx corrected for ejection duration and heart rate. Patients underwent assessment after they had been recumbent for a $5 \mathrm{~min}$ period of rest in the same position. Readings were recorded from the non-dominant arm.

\section{PPP Preparation}

PPP was generated by 2 step centrifuge, first at $1,700 \mathrm{~g}$ for $10 \mathrm{~min}$ before harvesting of plasma and at $20,000 \mathrm{~g}$ for $20 \mathrm{~min}$ to harvest PPP. The frozen samples were thawed at room temperature for $20 \mathrm{~min}$ before use.

\section{Flow Cytometry}

EMP quantification was performed by flow cytometry, the detailed methodology having been described previously [20]. In short, PPP was incubated with phycoerythrin-conjugated antihuman CD31 (BD Pharminogen 55546, BD Biosciences, New Jersey, USA), allophycocyanin-conjugated antihuman CD42b (BD Pharminogen 551061, BD Biosciences, New Jersey, USA) and efluor450 Annexin-V marker (Annexin V Apoptosis Detection Kit eBioscience, UK), with the addition of $10 \mu \mathrm{m}$ diameter counting beads (Flow-Count ${ }^{\mathrm{TM}}$ Fluorospheres; Beckman Coulter, UK). Flow cytometry was performed using the Beckman Coulter Cyan ADP flowcytometer and analysed using Summit version 4.3 software, with gates set to exclude artefact and beads. EMPs were considered microparticles (MPs) that were positive for CD31 and Annexin V but negative for CD42b (CD31+/AnnexinV+/CD42b- EMPs) and quantified as MPs per microlitres. A sample output of EMPs in the Summit software is found in Figure 1.

\section{Statistical Method}

Descriptive statistics is presented as mean \pm one SD for normally distributed continuous variables or otherwise median (range). Between-group differences in EMP count and patient characteristics between primary renal diseases were analysed using one-way analysis of variance or Kruskal-Wallis test for these parametric and nonparametric tests respectively. The study was not specifically powered to explain this analysis given that it has not previouslybeen described.

The relationship between baseline parameters (age, BP, biochemistry and AIx) and EMP count (baseline EMP and SEMP) was analysed using Spearman's correlation coefficient.

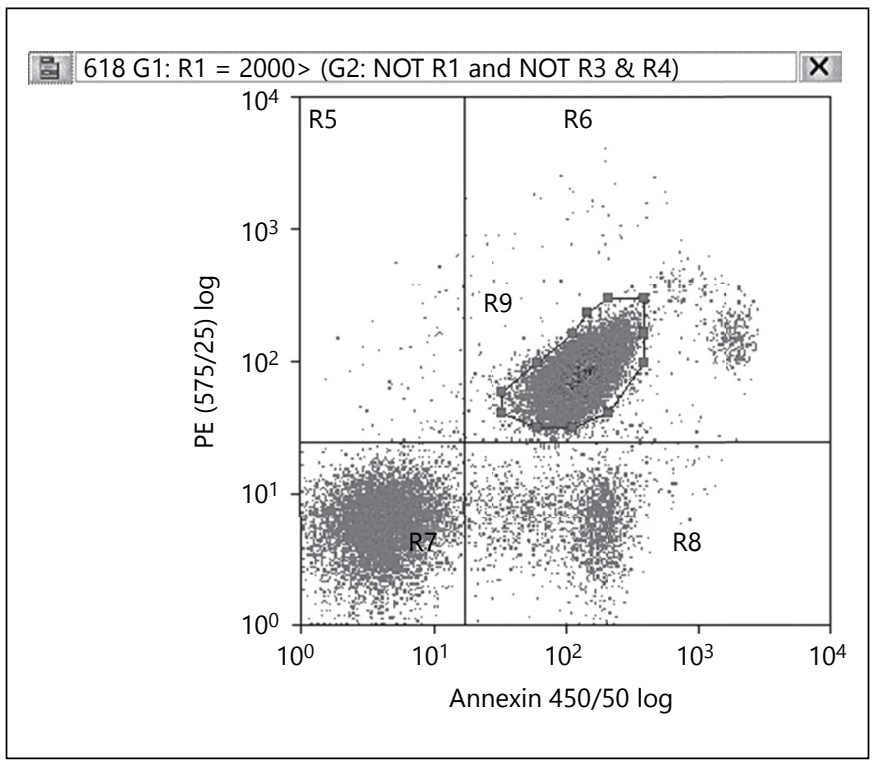

Fig. 1. Example of output for EMP in the Summit software.

Survival analyses were performed for time to (1) all-cause mortality, (2) major cardiovascular event or death, and (3) renal replacement therapy (dialysis or transplantation) for the study population as a whole. Follow-up time was from the date of collection of the second EMP sample. Cardiovascular events were defined as stroke, myocardial infarction, coronary artery intervention including bypass graft, non-coronary revascularisation, limb amputation for ischaemic injury, or hospitalisation for heart failure. Analyses used a multivariate Cox proportional hazard model. EMP values were log transformed and $\delta$ EMP values underwent affine and log transformation due to the high number of negative values. The model included EMP, $\delta \mathrm{EMP}$, and any other parameter noted to be significant on univariate analysis including demographics, co-morbidities, laboratory parameters and AIx. The hazard ratios (HRs) given for EMP and $\delta$ EMP are for unit change in $\log$ value. For this and all other analyses, statistical significance was set at $p<0.05$.

\section{Results}

\section{Baseline Characteristics}

There were 123 patients. The mean age was $63 \pm 11$ years, systolic BP $135 \pm 18 \mathrm{~mm} \mathrm{Hg}$ and eGFR $32 \pm 16 \mathrm{~mL} /$ $\mathrm{min} / 1.73 \mathrm{~m}^{2}$. The diagnoses were $\mathrm{cANCA}$ vasculitis $(n=$ $21)$, IgA nephropathy $(n=25)$, diabetic nephropathy $(n=$ $25)$ hypertension $(n=26)$, and ADPKD $(n=26)$. The overall median EMP count was $144 / \mu \mathrm{L}$ (range $10-714 / \mu \mathrm{L}$ ). There was a numerical but not statistical difference in EMP count between CKD diagnoses (Table 1; Fig. 2), with ADPKD being the highest (253 [41-610]) and cANCA vasculitis being the lowest (107 [21-610], $p=0.06)$. There 

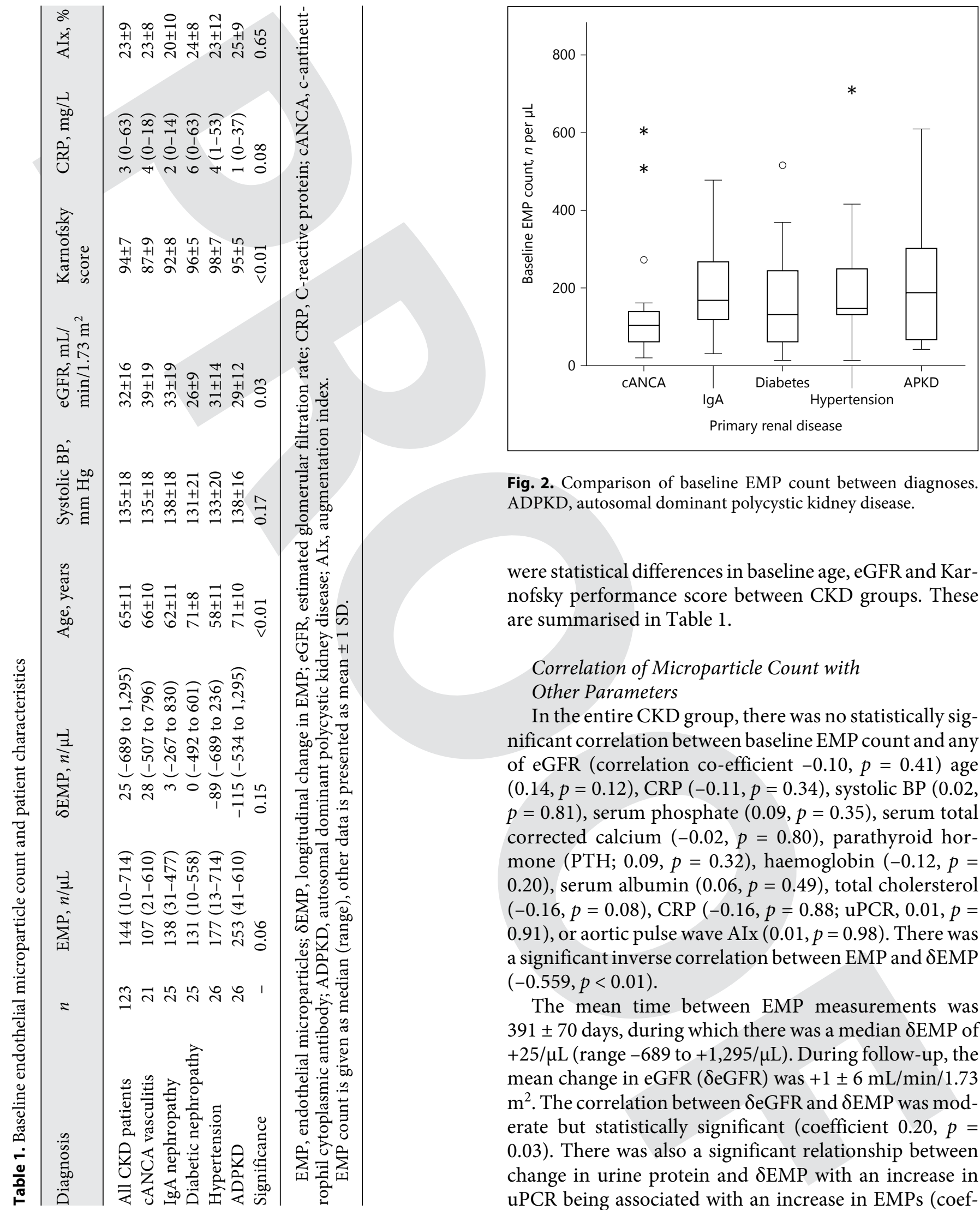

Fig. 2. Comparison of baseline EMP count between diagnoses. ADPKD, autosomal dominant polycystic kidney disease.

were statistical differences in baseline age, eGFR and Karnofsky performance score between CKD groups. These are summarised in Table 1.

\section{Correlation of Microparticle Count with}

Other Parameters

In the entire CKD group, there was no statistically significant correlation between baseline EMP count and any of eGFR (correlation co-efficient $-0.10, p=0.41$ ) age $(0.14, p=0.12)$, CRP $(-0.11, p=0.34)$, systolic BP $(0.02$, $p=0.81)$, serum phosphate $(0.09, p=0.35)$, serum total corrected calcium $(-0.02, p=0.80)$, parathyroid hormone (PTH; 0.09, $p=0.32)$, haemoglobin $(-0.12, p=$ $0.20)$, serum albumin $(0.06, p=0.49)$, total cholersterol $(-0.16, p=0.08)$, CRP $(-0.16, p=0.88$; uPCR, $0.01, p=$ $0.91)$, or aortic pulse wave $\operatorname{AIx}(0.01, p=0.98)$. There was a significant inverse correlation between EMP and $\delta \mathrm{EMP}$ $(-0.559, p<0.01)$.

The mean time between EMP measurements was $391 \pm 70$ days, during which there was a median $\delta$ EMP of $+25 / \mu \mathrm{L}$ (range -689 to $+1,295 / \mu \mathrm{L}$ ). During follow-up, the mean change in eGFR ( $\delta$ eGFR) was $+1 \pm 6 \mathrm{~mL} / \mathrm{min} / 1.73$ $\mathrm{m}^{2}$. The correlation between $\delta \mathrm{eGFR}$ and $\delta \mathrm{EMP}$ was moderate but statistically significant (coefficient $0.20, p=$ 0.03 ). There was also a significant relationship between change in urine protein and SEMP with an increase in uPCR being associated with an increase in EMPs (coef- 


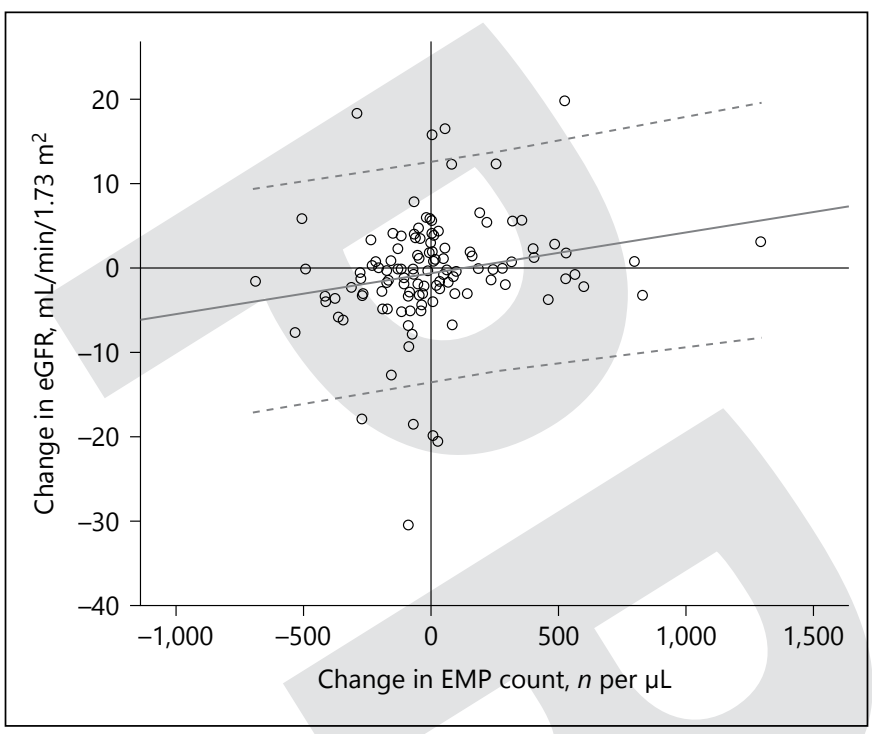

Fig. 3. Correlation between longitudinal change in eGFR and concurrent change in EMP count.

ficient $0.21, p=0.02$ ). The correlation between $\delta$ eGFR and $\delta E M P$ retained its significance after adjustment for change in uPCR (Fig. 3). There was no other significant correlation between longitudinal change in EMP count and change in any other measured laboratory parameter or AIx.

\section{Disease-Specific Correlations}

The relationship between change in EMP from baseline to follow-up and change in eGFR appeared to differ between diagnoses. For vasculitis and ADPKD, there were large positive correlations between $\delta \mathrm{EMP}$ and $\delta$ eGFR. The respective correlation coefficients for these diagnoses were $0.40(p=0.06)$ and $0.49(p<0.01)$. For diabetic nephropathy and IgA nephropathy, there were negative correlations: $-0.32(p=0.12)$ and $-0.24(p=$ $0.29)$, respectively. The coefficient in hypertension was $0.08(p=0.71)$.

The correlation between EMP and eGFR was not significant when analysed by each individual primary renal disease. There were very few disease-specific significant correlations between EMP and any baseline laboratory parameters or AIx, and between $\delta \mathrm{EMP}$ and longitudinal change in any parameter. Patients with IgA nephropathy demonstrated a significant correlation between $\delta \mathrm{EMP}$ and $\delta \mathrm{uPCR}$ (coefficient 0.557, $p<$ 0.01 ). Patients with hypertension demonstrated a correlation between EMP and age $(0.525, p<0.01)$ and uPCR $(0.509, p<0.01)$. Patients with hypertension also demonstrated a negative correlation between $\delta \mathrm{EMP}$ and both $\delta$ phosphate $(-0.398, p=0.04)$ and $\delta \mathrm{PTH}$ $(-0.520, p=0.02)$.

\section{Survival Analysis 1: All-Cause Mortality}

The mean follow-up period after the second EMP measurement was $3.9 \pm 2.2$ years. During this time, there were 32 deaths ( $26 \%$ of CKD patients). The leading causes of death were coronary artery disease $(n=11,34 \%$ of deaths) and pneumonia ( $n=7,22 \%)$. End-stage kidney disease, heart failure and malignancy were each responsible for 3 deaths (9\% each). On univariate analysis of allcause mortality, EMP was statistically associated with increased risk of death. The HR, for all-cause mortality with each unit increase in log EMP was 3.31 (95\% CI 1.129.76, $p=0.03)$. $\delta \mathrm{EMP}$ was not significantly associated (HR 0.64 [0.21-1.94], $p=0.43$ ). Other parameters that were positive on univariate analysis were age, diabetes, Karnofsky score, eGFR, PTH, CRP, and haemoglobin. The results for AIx were not significant (HR 1.02 [0.971.07]). Full results of the univariate analyses are found in Table 2. In the multivariate Cox regression analysis of allcause mortality, EMP retained its significance (HR 8.20 [1.67-40.2]), as did age and eGFR. In this model, $\delta$ EMP showed a numerical association with mortality, but this did not reach significance (HR 2.69 [0.04-165], $p=0.64$ ). The full results of the multivariate model are found in Table 3, alongside goodness of fit results for the multivariate model.

Because of the strong inverse correlation between EMP and $\delta E M P$ noted above (i.e., those patients with the highest baseline EMPs saw the greatest numerical fall in EMPs), $\delta E M P$ was also analysed as a percentage change from baseline to follow-up sampling. In univariate analysis, the affine log-transformed percentage $\delta \mathrm{EMP}$ also failed to reach statistical significance (HR 0.81 [0.41$1.58], p=0.53)$.

\section{Survival Analysis 2: Cardiovascular Events}

There were 22 major cardiovascular events (18\%) including 9 cardiovascular deaths ( $28 \%$ of deaths, $7 \%$ of patients). Age was the only parameter that demonstrated an association with cardiovascular outcome on univariate analysis (HR 1.05 [1.00-1.09], $p=0.03$ ). The complete univariate model is found in Table 2. Neither EMP nor $\delta \mathrm{EMP}$ were significant. In the multivariate survival analysis, no parameters demonstrated statistical significance. Indeed, the goodness-of-fit analysis itself was non-significant in this model. The results of the multivariate analysis are found in Table 3. 
Table 2. Univariate association of all measured parameters with each of all-cause mortality, major cardiovascular events, and progression to renal replacement therapy

\begin{tabular}{|c|c|c|c|c|c|c|}
\hline & \multicolumn{2}{|l|}{ All-cause mortality } & \multicolumn{2}{|c|}{ Cardiovascular event } & \multicolumn{2}{|l|}{ RRT } \\
\hline & HR (95\% CI) & $p$ value & HR (95\% CI) & $p$ value & HR (95\% CI) & $p$ value \\
\hline Age, years & $1.11(1.07-1.16)$ & $<0.01$ & $1.05(1.00-1.09)$ & 0.03 & $0.99(0.96-1.02)$ & 0.49 \\
\hline Systolic BP, mm Hg & $1.00(0.98-1.02)$ & 0.93 & $1.00(0.98-1.02)$ & 0.97 & $1.00(0.98-1.02)$ & 0.71 \\
\hline Body mass index, $\mathrm{kg} / \mathrm{m}^{2}$ & $1.04(0.97-1.12)$ & 0.26 & $1.06(0.98-1.14)$ & 0.16 & $1.01(0.94-1.08)$ & 0.85 \\
\hline Diabetes & $2.44(1.17-5.07)$ & 0.02 & $2.15(0.91-5.10)$ & 0.08 & $1.21(0.54-2.71)$ & 0.65 \\
\hline Heart failure & $1.57(0.21-11.56)$ & 0.66 & $0.05(0.00-3519)$ & 0.59 & $0.05(0.00-459)$ & 0.51 \\
\hline Male gender & $0.72(0.34-1.49)$ & 0.37 & $1.23(0.50-3.06)$ & 0.65 & $0.69(0.34-1.40)$ & 0.30 \\
\hline Karnofsky score & $0.95(0.91-0.99)$ & 0.03 & $0.96(0.91-1.01)$ & 0.12 & $0.99(0.93-1.04)$ & 0.63 \\
\hline EMP count & $3.31(1.12-9.76)$ & 0.03 & $0.55(0.19-1.64)$ & 0.28 & $0.97(0.38-2.46)$ & 0.95 \\
\hline$\delta \mathrm{EMP}$ & $0.64(0.21-1.94)$ & 0.43 & $6.04(0.41-89.93)$ & 0.19 & $0.42(0.12-1.45)$ & 0.17 \\
\hline AIx, \% & $1.02(0.97-1.07)$ & 0.41 & $0.98(0.93-1.04)$ & 0.49 & $0.99(0.95-1.03)$ & 0.62 \\
\hline $\mathrm{eGFR}, \mathrm{mL} / \mathrm{min} / 1.73 \mathrm{~m}^{2}$ & $0.95(0.92-0.98)$ & $<0.01$ & $0.97(0.93-1.00)$ & 0.07 & $0.94(0.91-0.97)$ & $<0.01$ \\
\hline Phosphate, mmol/L & $1.95(0.50-7.64)$ & 0.33 & $2.11(0.41-10.76)$ & 0.37 & $6.05(1.38-26.4)$ & 0.02 \\
\hline Corrected calcium, $\mathrm{mmol} / \mathrm{L}$ & $1.94(0.12-31.74)$ & 0.64 & $0.42(0.01-15.73)$ & 0.64 & $0.53(0.03-10.1)$ & 0.67 \\
\hline $\mathrm{PTH}, \mathrm{pmol} / \mathrm{L}$ & $1.00(1.00-1.01)$ & 0.02 & $1.00(0.99-1.01)$ & 0.90 & $1.01(1.00-1.01)$ & $<0.01$ \\
\hline Albumin, $g / L$ & $0.98(0.90-1.06)$ & 0.57 & $0.99(0.89-1.11)$ & 0.91 & $0.89(0.83-0.96)$ & $<0.01$ \\
\hline Cholesterol, mmol/L & $0.67(0.44-1.02)$ & 0.06 & $1.19(0.77-1.83)$ & 0.44 & $1.00(0.70-1.42)$ & 0.99 \\
\hline CRP mg/L & $1.03(1.00-1.06)$ & 0.05 & $1.03(0.99-1.06)$ & 0.11 & $1.01(0.98-1.05)$ & 0.54 \\
\hline uPCR g/mol & $1.00(1.00-1.00)$ & 0.66 & $1.00(1.00-1.00)$ & 0.90 & $1.01(1.00-1.02)$ & $<0.01$ \\
\hline Haemoglobin g/L & $0.97(0.95-0.99)$ & 0.01 & $0.99(0.97-1.02)$ & 0.52 & $0.96(0.94-0.98)$ & $<0.01$ \\
\hline
\end{tabular}

$\mathrm{HR}$, hazard ratio; RRT, real replacement therapy; BP, blood pressure; EMP, endothelial microparticle; $\delta \mathrm{EMP}$, longitudinal change in EMP; AIx, augmentation index; PTH, parathyroid hormone; CRP, C-reactive protein; uPCR, urine protein:creatinine ratio; eGFR, estimated glomerular filtration rate.

Table 3. Multivariate models demonstrating the independent association of parameters with each of all-cause mortality, major cardiovascular events, and progression to renal replacement therapy

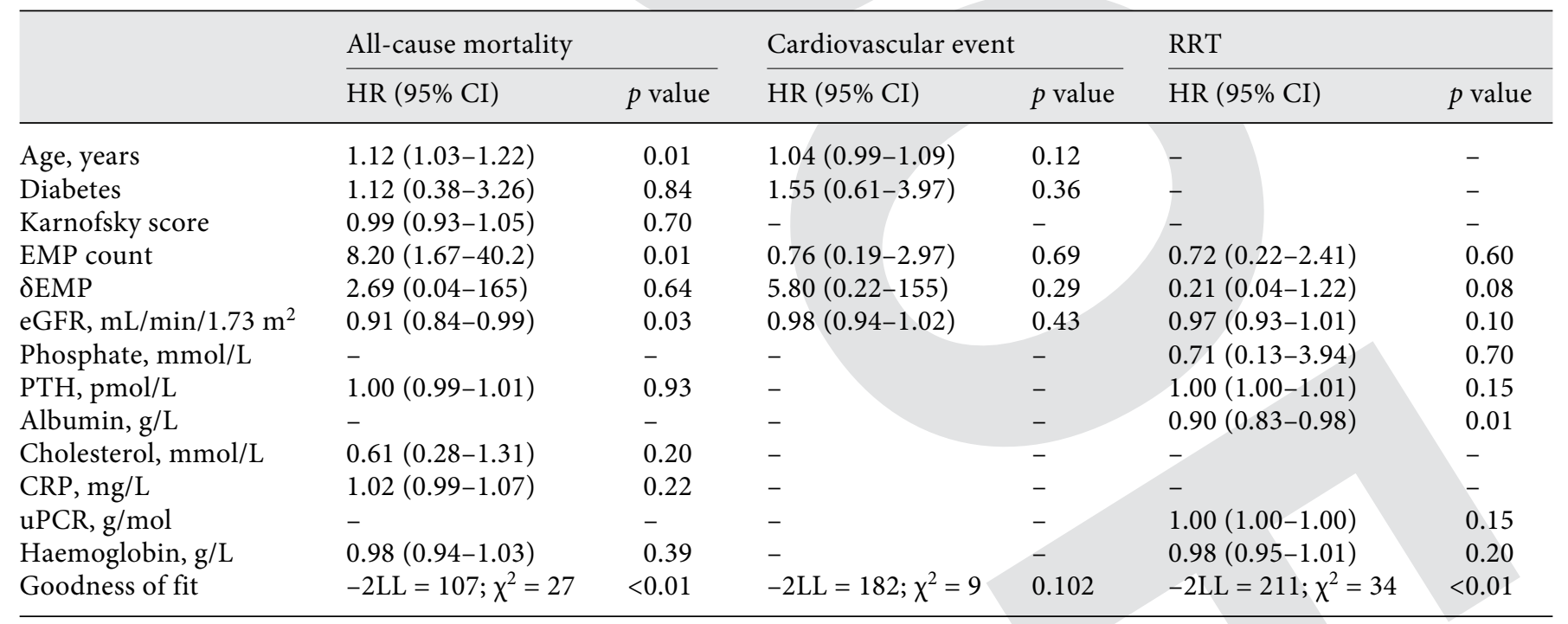

HR, hazard ratio; RRT, real replacement therapy; EMP, endothelial microparticle; $\delta \mathrm{EMP}$, longitudinal change in EMP; AIx, augmentation index; PTH, parathyroid hormone; CRP, C-reactive protein; uPCR, urine protein:creatinine ratio; $-2 \mathrm{LL},-2$ log likelihood; $\chi^{2}$, chi-square. 
Survival Analysis 3: Renal Replacement Therapy

Thirty-one patients required renal replacement therapy during the follow-up period (25\%). EMP and $\delta$ EMP were not significant on either univariate or multivariate analysis. The results of these analyses are found in Tables 2 and 3, respectively. The only independent predictor of disease progression to real replacement therapy in the multivariate model was serum albumin (HR 0.90 [0.83-0.98], $p=0.01$ ).

\section{Discussion}

\section{EMP in Different Renal Diseases}

In this study of patients with CKD but no prior cardiovascular or smoking history, there was a difference in baseline EMP count for different primary renal diagnoses with a $p$ value of $=0.06$. Of note, the 2 diseases with the highest median EMP count were ADPKD and hypertension. Although EMPs may be associated with an inflammatory state, shear stress is a key trigger to EMP release. In turn, hypertension will lead to intra-vascular shear stress. The lower eGFR in ADPKD compared to other primary diseases may also have been contributory, although there was no significant correlation between eGFR and EMP in this analysis. Furthermore, EMPs behave differently in different disease states and so their absolute values may be difficult to interpret in the context of renal function alone. For example, EMPs have been noted to be elevated in type 1 diabetes compared to controls but not in type 2 diabetes compared to controls in the same study [27]. EMPs have also been found in high levels in severe hypertension in patients without CKD compared to normal controls, as well as in acute coronary syndrome [16]. In these settings, their formation may be a response to, rather than a precursor of, vascular damage. This is supported by in vitro findings of EMPs promoting angiogenesis and thrombosis.

\section{Correlation of $\delta E M P$}

The positive correlation between $\delta$ EMP and $\delta$ eGFR (i.e., that EMP levels increased where eGFR improved over time) may seem surprising. However, this is not a unique finding. A previous study has shown higher serum levels of the vascular endothelial activation markers soluble intercellular adhesion molecule 1 (sICAM-1), sVCAM-1 and sE-selectin in both patients with pre-dialysis CKD and on dialysis when compared to normal eGFR controls but without a difference in level between CKD and dialysis groups [23].

There has also been no adjustment made for prescribed medication in our study. One may hypothesise that therapeutic interventions will affect EMP count independent of eGFR, and thereby possibly account for the increase in EMPs as eGFR improves. This is important given that different primary renal diagnoses will necessitate different therapeutic approaches.

The positive correlation between proteinuria and $\delta \mathrm{EMP}$ is likely to reflect that proteinuria is associated with disease activity in many causes of CKD, such as diabetic nephropathy, IgA nephropathy, vasculitis and also hypertension. In the disease-specific correlation analyses, the most significant correlations between proteinuria and EMPs were in IgA nephropathy and hypertension. Proteinuria is also associated with increased cardiovascular risk in $\mathrm{CKD}$, and the proposed mechanisms include its role as a potential marker of inflammation in disease; it is also associated with endothelial dysfunction. These mechanisms, particularly the latter, are also known to be associated with elevated EMPs in non-CKD disease.

The other noteworthy disease-specific correlation that was found was between $\delta \mathrm{EMP}$ and both $\delta$ phosphate and $\delta \mathrm{PTH}(-0.520, p=0.02)$. These relationships were negative, indicating that a rise in PTH and phosphate would be found with a concurrent fall in EMPs. Phosphate and PTH are markers of CKD mineral bone disorder and would be expected to be associated with vascular damage rather than a fall in EMPs. It may be that these findings represent a type 1 error in view of the large number of correlation analyses performed.

\section{The Association of EMP with AIx}

We found no correlation between baseline or longitudinal change in EMP and AIx. Contrary to this, a previous study of 44 maintenance haemodialysis patients found a significant association between cross-sectional measurements $(r=0.46, p<0.01)$ [15]. The fact that our study was in non-dialysis CKD may provide understanding of this, as clinically significant vascular stiffness becomes more pronounced with falling eGFR. Dursun et al. [24] did find a relationship between EMP and vascular stiffness in a cohort of CKD patients of which some were not on dialysis. However, these were paediatric patients and the assessment was by way of PWV. PWV is thought to be a better indicator than AIx of the diffuse medial calcification noted in vascular stiffness associated with CKD.

\section{Survival Analysis}

We found a significant independent association of baseline EMP with mortality in this CKD population. However, despite EMP being a marker of vascular dys- 
function, the significant association did not extend to cardiovascular events. Such an association is noted elsewhere, such as in predicting cardiovascular events and death in patients with established coronary artery disease, and also in patients on dialysis. In a study of 81 maintenance haemodialysis patients, each log increase in baseline EMP was associated with a 22 -fold increased risk of all-cause and 20 -fold increased risk of cardiovascular death [28]. Our study included patients with better preserved eGFR in whom vascular risk would be expected to be lower, and this may account for the difference in HR for all-cause mortality, and the non-significance in our analysis of cardiovascular death.

Our results demonstrate a striking variability in both baseline and longitudinal changes in EMP despite selecting patients with apparently stable renal disease. We observed potential differences in expected EMP count between renal diseases, as well as differences between diseases in the association of EMP and $\delta$ EMP with other factors such as proteinuria and eFGR. This is reflected in the very wide CIs for $\delta \mathrm{EMP}$ in the multivariate survival analyses and the lack of statistical significance despite encouraging numerical HR for all-cause mortality and cardiovascular events, which reflect the finding of other studies. Evidently, a far larger study would be required to be adequately powered to provide conclusive answers to whether EMPs are a useful marker of cardiovascular risk in early CKD and whether its utility will differ between causes of renal failure.

\section{Conclusions}

EMP are elevated in CKD, but this appears to differ between renal diseases. The broad range of baseline EMP counts and $\delta \mathrm{EMP}$ create large CIs for determining what is "normal" EMP activity in CKD. These factors will inhibit the generalised utility of EMP as a biomarker of disease activity and vascular risk in CKD. Greater evaluation of EMP activity in different renal diseases during the different phases of the disease and at different stages of CKD is required.

\section{Acknowledgements}

We would like to thank the patients of Salford Royal Foundation Trust for their participation in this study.

\section{Disclosure Statement}

B.P. is supported by the NIHR Manchester Musculoskeletal Biomedical Research Unit and the NIHR Manchester Clinical Research Facility. This report includes independent research supported by (or funded by) the National Institute for Health Research Manchester Musculoskeletal research Unit. The views expressed in this publication are those of the author(s) and not necessarily those of the NHS, the National Institute for Health Research or the Department of Health.

\section{Statement of Ethics}

Ethical approval was granted by the relevant local ethics committee and the study complied with the Declaration of Helsinki.

\section{References}

1 Stack AG, Bloembergen WE: Prevalence and clinical correlates of coronary artery disease among new dialysis patients in the United States: a cross-sectional study. J Am Soc Nephrol 2001;12:1516-1523.

2 Foley RN, Parfrey PS, Sarnak MJ: Epidemiology of cardiovascular disease in chronic renal disease. J Am Soc Nephrol 1998;9(12 suppl):S16-S23.

3 Green D, Ritchie JP, Kalra PA: Meta-analysis of lipid-lowering therapy in maintenance dialysis patients. Nephron Clin Pract 2013;124: 209-217.

4 Green D, Panayotova R, Ritchie JP, O'Riordan E, McDonald J: Lipid-lowering therapy in chronic kidney disease: is there a role for ezetimibe? J Ren Care 2012;38:138146.

5 Goodman WG, Goldin J, Kuizon BD, Yoon C, Gales B, Sider D, et al: Coronaryartery calcification in young adults with end-stage renal disease who are undergoing dialysis. N Engl J Med 2000;342:14781483

6 Yao Q, Pecoits-Filho R, Lindholm B, Stenvinkel $\mathrm{P}$ : Traditional and non-traditional risk factors as contributors to atherosclerotic cardiovascular disease in end-stage renal disease. Scand J Urol Nephrol 2004;38:405416.

7 Eddington H, Hoefield R, Sinha S, Chrysochou C, Lane B, Foley RN, Hegarty J, New J, O'Donoghue DJ, Middleton RJ, Kalra PA: Serum phosphate and mortality in patients with chronic kidney disease. Clin J Am Soc Nephrol 2010;5:2251-2257.

8 Eddington $\mathrm{H}$, Kalra PA: The association of chronic kidney disease-mineral bone disorder and cardiovascular risk. J Ren Care 2010; 36(suppl 1):61-67.

9 Lee BT, Ahmed FA, Hamm LL, Teran FJ, Chen CS, Liu Y, Shah K, Rifai N, Batu- man V, Simon EE, He J, Chen J: Association of C-reactive protein, tumor necrosis factor-alpha, and interleukin-6 with chronic kidney disease. BMC Nephrol 2015; 16:77.

10 Schiro A, Wilkinson FL, Weston R, Smyth JV, Serracino-Inglott F, Alexander MY: Elevated levels of endothelial-derived microparticles, and serum CXCL9 and SCGF- $\beta$ are associated with unstable asymptomatic carotid plaques. Sci Rep 2015;5:16658.

11 Pitha J, Králová Lesná I, Stávek P, Mahrová A, Racek J, Sekerková A, Teplan V, Štollová M: Effect of exercise on markers of vascular health in renal transplant recipients. Physiol Res 2015;64:945-949.

12 Prochazka M, Procházková J, Lubušký M, Pilka R, Úlehlová J, Michalec I, Polák P, Kacerovský M, Slavik L: Markers of endothelial activation in preeclampsia. Clin Lab 2015;61: $39-46$. 
13 Heathfield SK, Parker B, Zeef LA, Bruce IN, Alexander MY: Certolizumab pegol attenuates the pro-inflammatory state in endothelial cells in a manner that is atheroprotective. Clin Exp Rheumatol 2013;31:225-233.

14 Schiro A, Wilkinson FL, Weston R, Smyth JV, Serracino-Inglott F, Alexander MY: Endothelial microparticles as conveyors of information in atherosclerotic disease. Atherosclerosis 2014;234:295-302.

15 Amabile N, Guérin AP, Leroyer A, Mallat Z, Nguyen C, Boddaert J, London GM, Tedgui A, Boulanger CM: Circulating endothelial microparticles are associated with vascular dysfunction in patients with end-stage renal failure. J Am Soc Nephrol 2005;16:3381-3388.

16 Sinning JM, Losch J, Walenta K, et al: Circulating CD31+/Annexin V+ microparticles correlate with cardiovascular outcomes. Eur Heart J 2011;32:2034-2041.

17 Preston RA, Jy W, Jimenez JJ, Mauro LM, Horstman LL, Valle M, Aime G, Ahn YS: Effects of severe hypertension on endothelial and platelet microparticles. Hypertension 2003;41:211-217.

18 González-Quintero VH, Jiménez JJ, Jy W, Mauro LM, Hortman L, O'Sullivan MJ, Ahn Y: Elevated plasma endothelial microparticles in preeclampsia. Am J Obstet Gynecol 2003; 189:589-593.
19 Agouni A, Lagrue-Lak-Hal AH, Ducluzeau $\mathrm{PH}$, MostefaI HA, Draunet-Busson C, Leftheriotis G, Heymes C, Martinez MC, Andriantsitohaina R: Endothelial dysfunction caused by circulating microparticles from patients with metabolic syndrome. Am J Pathol 2008; 173:1210-1219.

20 Parker B, Al-Husain A, Pemberton P, Yates AP, Ho P, Gorodkin R, Teh LS, Alexander MY, Bruce IN: Suppression of inflammation reduces endothelial microparticles in active systemic lupus erythematosus. Ann Rheum Dis 2014;73:1144-1150.

21 Faure V, Dou L, Sabatier F, Cerini C, Sampol J, Berland Y, Brunet P, Dignat-George F: Elevation of circulating endothelial microparticles in patients with chronic renal failure. J Thromb Haemost 2006;4:566-573.

22 Mezzano D, Tagle R, Pais E, Panes O, Pérez M, Downey P, Muñoz B, Aranda E, Barja P, Thambo S, González F, Mezzano S, Pereira J: Endothelial cell markers in chronic uremia: relationship with hemostatic defects and severity of renal failure. Thromb Res 1997;88: 465-472.

23 Bonomini M, Reale M, Santarelli P, Stuard S, Settefrati N, Albertazzi A: Serum levels of soluble adhesion molecules in chronic renal failure and dialysis patients. Nephron 1998;79: 399-407.
24 Dursun I, Poyrazoglu HM, Gunduz Z, et al: The relationship between circulating endothelial microparticles and arterial stiffness and atherosclerosis in children with chronic kidney disease. Nephrol Dial Transplant 2009;24:2511-2518.

25 Hoefield RA, Kalra PA, Baker P, et al: Factors associated with kidney disease progression and mortality in a referred CKD population. Am J Kidney Dis 2010;56:10721081.

26 Alderson HV, Ritchie JP, Middleton R, et al: FGF-23 and Osteoprotegerin but not Fetuin$A$ are associated with death and enhance risk prediction in non-dialysis chronic kidney disease stages 3-5. Nephrology (Carlton) 2016; 21:566-573.

27 Sabatier F, Darmon P, Hugel B, Combes V, Sanmarco M, Velut JG, Arnoux D, Charpiot P, Freyssinet JM, Oliver C, Sampol J, Dignat-George F: Type 1 and type 2 diabetic patients display different patterns of cellular microparticles. Diabetes 2002;51: 2840-2845.

28 Amabile N, Guérin AP, Tedgui A, Boulanger CM, London GM: Predictive value of circulating endothelial microparticles for cardiovascular mortality in end-stage renal failure: a pilot study. Nephrol Dial Transplant 2012;27: $1873-1880$ 